\title{
INTERACTIVIDAD EN LA RED CON ACTIVIDADES CABRI
}

\author{
Jesús Murillo Ramón \\ Josep M. Fortuny Aymemi \\ Universidad de La Rioja \\ Universidad Autónoma de Barcelona
}

\begin{abstract}
RESUMEN: El artículo que se presenta, tiene dos partes diferenciadas. En la primera se describe lo que constituye una innovación metodológica. El diseño y utilización de una infraestructura y soporte instruccional, constituida fundamentalmente por una red electrónica, Internet, un foro de discusión y el software de geometría CABRI II. En la segunda con los planteamientos e instrumentos diseñados, se analiza, estudia y evalúa la naturaleza de las interacciones que aparecen y los posibles beneficios que se generan, cuando un grupo de cinco alumnos desarrollan trabajo colaborativo en la resolución de actividades.
\end{abstract}

ABSTRACT: This paper is divided into two different parts. The first one describes a methodological innovation: the design and use of an educational infrastructure and support system basically composed of an electronic network, i.e., the Internet, a discussion forum and the geometry software CABRI II. Then, with the approaches and tools developed, the second part analyses, studies and evaluates the nature of the interactions and the potential benefits generated when five students cooperate to solve activities.

PALABRAS CLAVE: Aprendizaje autónomo, trabajo colaborativo, corresponsabilidad, beneficios cognitivos, interacción social, entornos virtuales de aprendizaje con Software de Geometría Dinámica.

KEYWORDS: Autonomous learning, collaborative work, co-responsibility, cognitive benefits, social interaction, virtual learning environment with Dynamical Geometry Software.

\section{OBJETIVOS Y CONTEXTO}

Pretendemos mejorar el proceso educativo en general y en particular el de las matemáticas de la E.S. para conseguir un mayor éxito escolar, mediante la utilización de sistemas interactivos y colaborativos de enseñanza, cuando los alumnos trabajan de manera flexible, bien de forma autónoma o en inter acción con el profesor u otros compañeros, potenciando a la vez el aprendizaje autónomo del alumno y la adqui- 
sición de actitudes, valores y hábitos formativos que pueda utilizar en un futuro en el mundo laboral.

Parece que el diseño y utilización de entornos interactivos que promuevan un cambio cultural en el mundo educativo, potenciando una enseñanza más centrada en el alumno, favorecen los aspectos considerados anteriormente

Por otra parte, no basta con potenciar el uso de entornos interactivos, es necesario también clasificar, estudiar y evaluar las interacciones a distancia y sus efectos en el aprendizaje a través de categorías de acciones e indicadores que permitan establecer la efectividad de las mismas cuando se desarrolla trabajo colaborativo.

Por tanto además de diseñar un entorno interactivo de aprendizaje, analizamos las interacciones que se producen y se determinan los aspectos beneficiosos de las TIC al integrarlas en el proceso educativo de las matemáticas.

\section{INFRAESTRUCTURA Y SOPORTE INSTRUCCIONAL}

La infraestructura técnica utilizada está constituida por una red local de ordenadores, que se encuentran conectados al servidor de la Universidad de la Rioja mediante una conexión de banda ancha, Los alumnos participantes disponen de una cuenta abierta en el servidor. Con su contraseña pueden acceder a las actividades que se plantean en la página principal así como al foro de discusión (Tablero electrónico) que se ha diseñado con software de dominio público, está escrito en Perl y creados por Matt Wright y se puede acceder entre otros en la dirección:

http://worldwidemart.com/scripts (http://www.scriptarchive.com/)

Los alumnos disponen asimismo como ayuda para resolver las cuestiones de Geometría del software de geometría CABRI II

\section{ECOSISTEMA DE APRENDIZAJE}

En nuestro caso, definimos ecosistema de aprendizaje, como un entorno interactivo, constituido por: los alumnos, el contenido/saber, el profesor/tutor y el medio, cuyos procesos de funcionamiento se relacionan entre sí y se desarrollan en función de los factores físicos de un mismo ambiente. De forma que la modificación de alguno de sus componentes modifica el estado de los restantes. A través del juego interactivo, con sus iguales o con el resto de los elementos del ecosistema, emerge la identidad personal del alumno.

Las distintas relaciones entre los diversos elementos del ecosistema las estudiamos utilizando como instrumentos dos planos y tres pirámides, que definimos a continuación.

Plano intersubjetivo o polígono sociograma, es un polígono en el que cada uno de sus vértices representa a cada uno de los alumnos que intervienen en la actividad. Sus aristas y diagonales representan las interacciones entre cada uno de los interactuantes (en el gráfico que se adjunta no están dibujadas todas, por una mejor claridad del mismo)

Plano de situación o triángulo de situación, es un triángulo en el que sus vértices representan respectivamente al profesor/tutor, al medio y al contenido/saber. Los lados representan las relaciones e influencias mutuas entre los vértices.

La pirámide roja representa la interactividad entre los alumnos y el medio. 

do.

La pirámide amarilla representa la interactividad entre los alumnos y el conteni-

La pirámide verde representa la interactividad ente los alumnos y el profesor

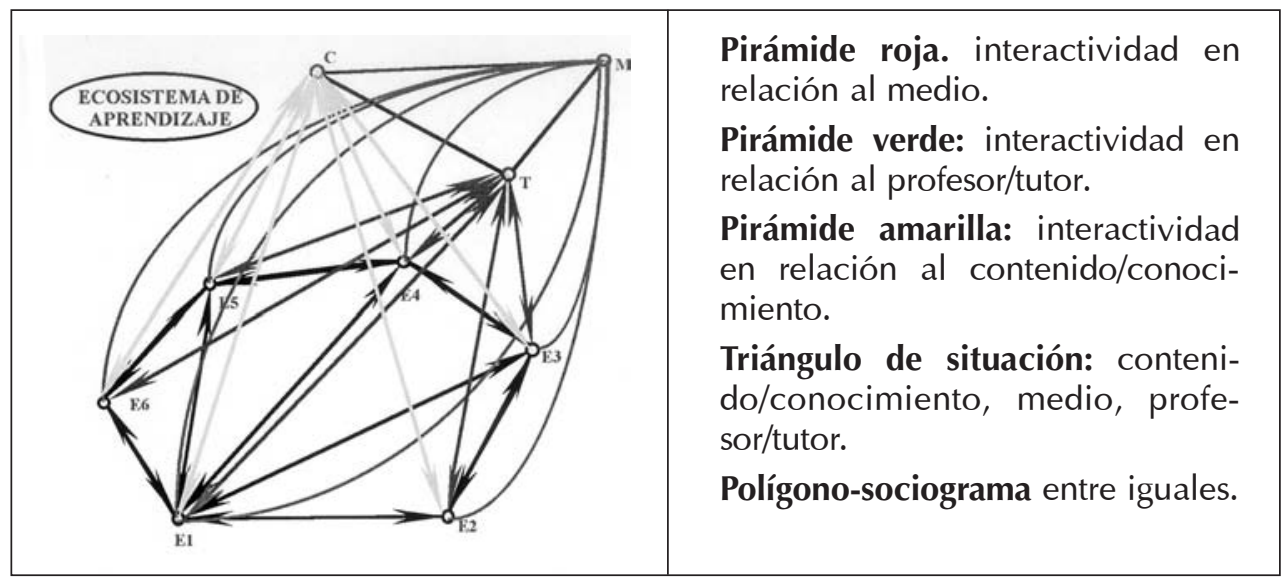

Figura 1. Ecosistema de aprendizaje

Pasamos a describir los dos planos antes citados

3.1. Plano intersubjetivo

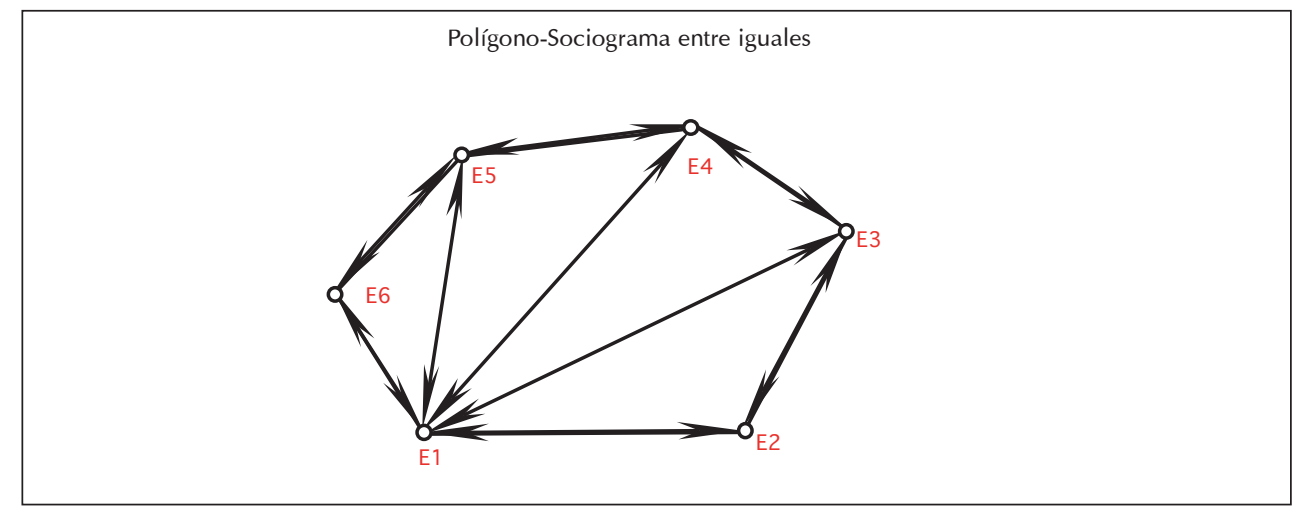

Figura 2. Polígono-Sociograma entre iguales

En este plano se producen las acciones e interacciones entre el modo de pensar y actuar de los alumnos, que denominamos relaciones entre iguales. Evidentemente el número de vértices del polígono sociograma, no siempre será el mismo, dependiendo en cada caso del número de alumnos que participan en la actividad que en cada momento se desarrolla y por supuesto también variará el número de interacciones -representadas por aristas y diagonales-. Los criterios que hemos asumido para elegir la muestra de alumnos han sido: Ser alumnos activos y con un cierto 
grado de afinidad -ha habido réplicas entre ellos-, distintos niveles académicos de los alumnos -a partir de la información del profesor presencial- y con distinto grado de participación en la clase presencial.

Pretendemos establecer un modo colaborativo de resolución de problemas entre profesor y alumnos, tomando en consideración las ideas del andamiaje (Bruner y sus colaboradores, 1975). Éstos acuñaron el término de andamiaje (scafolding) que fue conformado con las ideas teóricas de Vygostky -las ideas teóricas de Vigostky son muy anteriores, aunque Ilegaron bastante tarde al mundo occidental (Vigostky, L. S., 1978. Mind in Society. Cambridge: Harvard University Press)- que consideraba que las interacciones guiadas por adultos pueden ayudar a los niños a desarrollar funcionamiento psicológico superior y que la asistencia de un adulto permite al niño operar en la "zona de desarrollo próximo" (Álvarez y otros, 1997), área situada entre el nivel de lo que un niño puede conseguir por sí solo y el nivel que el mismo niño puede alcanzar con asistencia. El andamiaje involucra al profesor que realiza parte de la tarea que el estudiante todavía no puede manejar y le proporciona soporte que se modifica gradualmente hasta que los alumnos se hacen cargo de su aprendizaje sin la ayuda del profesor.

Para determinar el papel del alumno en las interacciones establecemos una serie de categorías, teniendo en cuenta las relaciones entre iguales, las relaciones con el medio y las relaciones del alumno con el saber.

\subsection{PLANO DE SITUACIÓN}

En él tienen lugar las acciones e interacciones entre el profesor/tutor, el contenido/conocimiento y el medio, representados respectivamente por cada uno de los vértices del triángulo.

Triángulo de situación. Vértices: contenido/conocimiento, medio profesor/tutor

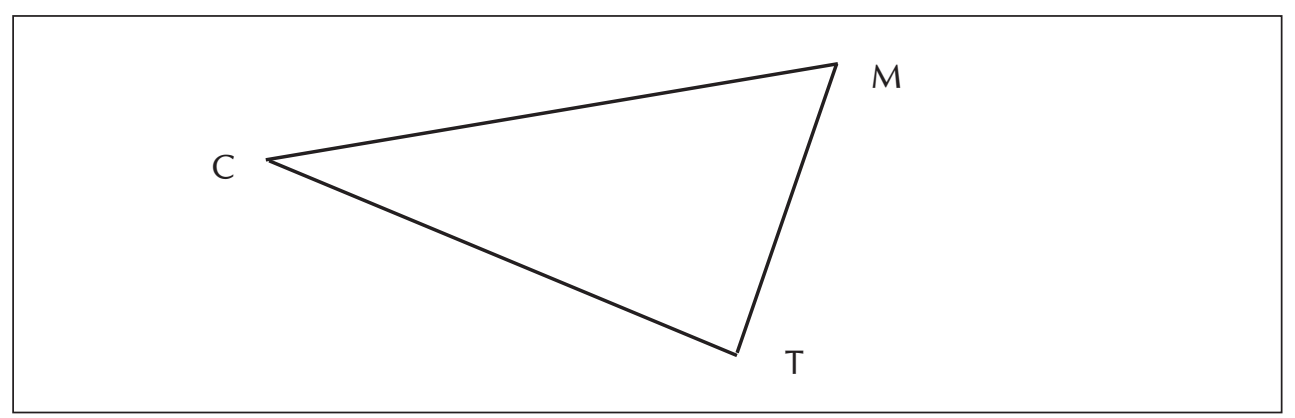

Figura 3. Triángulo de situación

C: Algunos objetivos generales, en relación con la formación geométrica, parecen claros y se consideran necesarios para nuestros alumnos al finalizar la educación obligatoria -incorporar al lenguaje y modos de argumentación habituales la expresión geométrica, a fin de comunicarse de manera rigurosa y precisa, utilizar los conocimientos...). Otro asunto, un poco más delicado y complejo es qué contenidos geométricos concretos se deben seleccionar. 
A la hora de planificar las actividades geométricas (C.I.P.), 1996) con CABRI puede ser de gran utilidad establecer una categorización de las mismas (Murillo, J, 2001).

Las actividades con CABRI se organizan de manera que interesen a los alumnos y les permitan participar en ellas.

T: Desarrollar y aplicar nuevos métodos de aprendizaje para generar interacciones más efectivas, supone replantearse el papel del profesor y que cada uno reflexione sobre su papel en el proceso educativo. Consideramos que la función fundamental del profesor/tutor sería la de intentar modular y guiar la discusión de los estudiantes, facilitando el aprendizaje y ayudándoles a lo largo de todo el proceso. El discurso instruccional se transforma en diálogo y el papel del profesor consiste por tanto en apoyar el aprendizaje.

M: Entorno rico: Las tareas a desarrollar para conseguir el conocimiento que se pretende, son situaciones-problema abiertas, con múltiples fases y en las que en su resolución se utilizan medios informáticos. Con la terminología utilizada por R. Hershkowitz (Hershkowitz, 1999) a un tal entorno, que cumple las anteriores características lo llamamos "enviroment rich".

Los entornos informáticos permiten a sus usuarios manipular de una forma más directa los objetos matemáticos y sus relaciones, concretando de alguna manera los conceptos matemáticos abstractos. Lo que los diferencia de otros materiales pedagógicos, es su naturaleza intrínsecamente cognitiva (Balachef y Kaput, 1997). Hay un cambio de complejidad entre la manipulación de un instrumento material y la utilización de software, debido no sólo al número o a la velocidad del número de operaciones posibles. Por un ejemplo, la utilización de Cabri es totalmente diferente de la de la regla y compás. Diferencia que estriba fundamentalmente en que los entornos de aprendizaje como Cabri, manipulan el conocimiento geométrico de una forma totalmente distinta al de la regla. Estos entornos de aprendizaje, se construyen a partir de una representación del conocimiento para un sistema de objetos y relaciones, representación accesible al usuario en la interacción de una forma más o menos significativa en relación al conocimiento (Balacheff, 1994). El aprendizaje es el resultado de una construcción de conocimientos en el curso de la interacción con el entorno. El trabajo realizado con el conocimiento para que resulte manejable por el sistema-transposición informática- determina fuertemente la interacción entre el usuario y el sistema y por tanto el conocimiento que emerge de esta interacción (Balacheff, 1994).

\section{ROL DEL PROFESOR EN LA INTERACCIÓN}

Cuando la escuela se organiza como "comunidad de aprendizaje", las actividades se organizan de forma que interesen a los alumnos y les permitan participar en ellas, comprendiendo la finalidad de las mismas. El discurso instruccional se transforma en diálogo y el papel del profesor consiste en apoyar el aprendizaje y no en controlar las interacciones en el aula. De esta manera la clase se convierte en una organización compleja, en la que sus miembros deben aprender a asumir la responsabilidad de su propio aprendizaje; todos trabajan y en función del grado de comprensión de su actividad, asumen papeles diferentes y diversos grados de responsabilidad. 


\subsection{CATEGORÍAS PROFESOR/TUTOR-MEDIO}

El profesor como gestor del trabajo debe llevar a cabo como mínimo las siguientes categorías de actividades: de organización, interrogación, explicación, valoración y advertencia (tomado de Gairín, J.M, 1998). El análisis de las mismas permite estudiar la forma en que se ha organizado la enseñanza, de tener los datos de cómo funciona el sistema que se ha diseñado; conociendo de esta manera si el medio y la organización que le acompaña son correctos o presentan deficiencias. Esta clasificación de categorías permite controlar las interacciones entre las variables profesor/tutor y medio.

PO: El profesor ORGANIZA. Su responsabilidad como gestor del trabajo en la Comunidad Virtual de Aprendizaje le obliga a llevar a cabo diferentes tipos de actuaciones: dirigir la gestión, reclamar la atención de los estudiantes...

PP: El profesor PREGUNTA. Necesita conocer la opinión de los estudiantes en relación con la gestión de Comunidad Virtual de Aprendizaje: ¿El sistema funciona bien?, ¿hay dificultades en la utilización de los programas?, ¿las respuestas llegan en el tiempo adecuado...?

PE: El profesor EXPLICA. Tiene que transmitir a los alumnos sus decisiones sobre la gestión: Para enviar un mensaje con el correo electrónico es necesario..., las respuestas tienen que llegar en un mensaje de correo con el archivo de CABRI como documento adjunto, responder en el Tablero Electrónico...

PV: El profesor VALORA. Las actuaciones que se producen en el desarrollo de alguna actividad pueden ser cuestionadas por los alumnos $y$, en consecuencia, el profesor debe atender a sus razones: Hola Cristian: Gracias por el aviso en relación con las actividades. He recibido tus respuestas a las cuestiones planteadas en la actividad A8 y decirte que son correctas. Saludos: Jesús

PA: El profesor ADVIERTE. Tiene que juzgar las actuaciones de los alumnos y tomar decisiones al respecto: observo que no enviáis los mensajes por el procedimiento que se indicó, por favor escribir el texto explicativo en el archivo de Cabri...

\subsection{CATEGORÍAs PROFESOR/TUtOR-AlumnO}

En cuanto a la relaciones del profesor con los alumnos, establecemos las siguien tes categorías de actividades de actuación cuando se dirige a los alumnos en relación con los contenidos:

$$
\text { explorar, aclarar, valorar, proponer, sistematizar... }
$$

En cuanto al diseño y a la forma en cómo se presentan los contenidos a los alumnos establecemos las siguientes categorías:

$$
\text { organizar, preguntar, concretar, valorar e intervenir. }
$$

Categorías, que nos permitirán analizar la propuesta que el profesor hace a los alumnos -curriculum implementado-.

Las acciones alumno-profesor en la comunicación multidireccional casi no se han dado ya que en este tipo de comunicación el profesor es considerado un igual por lo que este tipo de acciones deberían ser consideradas dentro de las categorías entre iguales.

El análisis de las acciones del profesor cuando se dirige a los alumnos en relación con los contenidos, nos permite establecer una serie de categorías que caracterizan las interacciones del profesor con los alumnos: 
EXPLORA. El profesor explora significados para conocer las percepciones y concepciones de los alumnos

Hola Cristian: En la solución 2, para que el rombo obtenido tenga la misma área que el rombo inicial, ¿se tienen que dar unas determinadas condiciones en cuanto a alguno de sus elementos?, ¿se verifica para cualquiera? Razona tu respuesta e intenta dar un teorema que recoja las condiciones en las que se cumple o no esta situación.

Saludos

PEX:

CLARIFICA. Con la pregunta que os planteaba en relación al rombo y al cuadrado, casi con toda seguridad por no haberla hecho con suficiente claridad, ha habido diversas interpretaciones que han aportado muy variadas respuestas, y muchas de ellas realmente creativas y originales. Os la envio de nuevo con alguna modificación que espero os permita una mejor comprensión de la misma.

PCL

ACLARA. Hola Cristian: Tus respuestas en relación con la actividad A9 son correctas, señalarte sólo algún matiz: Cuidado con HALLA y HAYA. ¿Podrías expresar se una forma un poco más completa la obtención de los centros de la homotecia, en función de elementos homólogos, correspondientes o imágenes? PCA

VALORA. Tus aclaraciones me han servido perfectamente. PVA

PROPONE. Creo que es interesante seguir, pues aparecen propiedades de los cuadriláteros y triángulos que justifican las posibles respuestas.

PPR

ANIMA. En la cuestión relativa al rombo y al cuadrado, posiblemente no me he expresado con suficiente claridad, por lo que ha habido diversas interpretaciones que han aportado muy variadas respuestas, y muchas de ellas realmente creativas y originales. En cualquier caso quiero seguir insistiendo un poquito, planteándola de nuevo, ligeramente modificada y os animo a seguir trabajando. PAN

SISTEMATIZA. Tus respuestas y los argumentos dados en las tres cuestiones planteadas en el problema1 son correctas.

PSI

\subsection{CATEGORÍAS PROFESOR/TUTOR-CONTENIDO.}

En cuanto al diseño y a la forma en cómo se presentan los contenidos a los alumnos establecemos las siguientes categorías: organizar, preguntar, concretar, valorar e intervenir, que nos permitirán analizar la propuesta que el profesor hace a los alumnos -curriculum implementado-.

\section{PAPEL DE ALUMNO EN LA INTERACCIÓN}

Nos planteamos, conseguir la participación activa de los alumnos en su aprendizaje y en la construcción del conocimiento. Para analizar el papel del alumno en las interacciones establecemos una serie de categorías, considerando las relaciones entre iguales, las relaciones con el medio y las relaciones del alumno con el saber.

\subsection{CATEGORÍAS ENTRE IGUALES}

ACA. Un alumno SOLICITA aclaraciones a otro alumno. Solicitando aclaraciones se fomenta tanto la observación de las dificultades de comprehensión como el uso 
de estrategias de reprocesamiento, tales como una búsqueda selectiva de contenido relevante y relectura. Ilustramos esta categoría con:

Re: Problema 3, enviado por Eduardo, en contestación a: Problema 3 enviado por Cristian

: ¿Y de donde salen los tres triángulos?

ACL. Un alumno CLARIFICA aspectos a otro. Un alumno actúa para facilitar la comprensión de algún tipo de contenido a otro compañero.

Re: Problema 3, enviado por Cristian, en contestación a: Re: Problema 3 enviado por Eduardo

Salen de la unión del punto $P$ con los vértices del triangulo grande.

AVA: Un alumno VALORA los resultados obtenidos por otro compañero. Informa a su compañero sobre su opinión en relación con la calidad del proceso seguido o del resultado obtenido.

Re: Problema 3, enviado por Eduardo, en contestación a: Re: Problema 3 enviado por Cristian.

: Salen de la unión del punto $\mathrm{P}$ con los vértices del triangulo grande.

: En este caso ya está todo bastante claro, solo decirte que se entiende mejor poniéndolo con letra las formulas.

AAA: Un alumno ANIMA a otro alumno. Ante una situación de bloqueo o respuesta pertinente pero incompleta un alumno da ánimos a otro compañero para continuar.

Re: Problema 3, enviado por Oscar, en contestación a: Re: Problema 3 enviado por Diego

Yo no busco ninguna explicación, lo que pasa es que te estoy dando un consejo de cómo estaría mejor.

ACI: Un alumno realiza comentarios no pertinentes a la actividad considerada.

Re: Problema 3, enviado por Oscar, en contestación a: Re: Problema 3 enviado por Diego

Quitate el polvo. to.

ANA: Un alumno NO ACEPTA sugerencias de otros y mantiene su planteamien-

Re: Problema 3, enviado por Diego, en contestación a: Re: Problema 3 enviado por Oscar

:Como voy ha mejorarlo si es perfecto -se ha respetado la respuesta textual del alumno-

\subsection{CATEGORÍAS ALUMNO/MEDIO}

Las siguientes categorías, caracterizan las acciones de los alumnos en relación a su opinión sobre el funcionamiento del sistema que se ha diseñado y de la organización que lo acompaña y que nos permitirán obtener datos sobre el comportamiento correcto del sistema y proceder en su caso a una mejora del mismo.

El tipo de acciones puestas de manifiesto por los mensajes electrónicos relativas al comportamiento del sistema ha sido mínimo. Por escrito, acciones del tipo suge- 
rencia, preguntas, explicaciones o valoraciones no aparecen. No obstante durante la fase cuasi experimental de la investigación si que se dieron. Podemos interpretar la ausencia de su manifestación por escrito con una doble justificación: Por una parte la mayoría de las cuestiones y aclaraciones de tipo técnico relacionadas con el sistema soporte son resueltas por el profesor presencial en el aula y por otra parte el sistema soporte en general funciona de forma bastante correcta.

AP: Los alumnos PREGUNTAN algún aspecto de tipo técnico o funcionamiento del sistema soporte. Quieren disponer de informaciones más amplias o concretas sobre la gestión.

\section{Actividad A8.}

From: "CRISTIAN “<cristian.@proys.unirioja.es> To: <jmurillo@dmc.unirioja.es>,

Hola: Le envio las respuestas a las cuestiones planteadas en la actividad A8 en el fichero de Cabri. Queria comentarle que en la pagina del proyecto solo aparecen activadas las cuatro primeras preguntas, por lo que faltan esta y todas las demas preguntas. Espero que se solucione y podamos continuar con la actividad A9.

\section{Un saludo: Cristian}

AE: Los alumnos EXPLICAN. Dan información sobre sus actuaciones o sus propuestas sobre la gestión.

\section{Actividad A8.}

From: "CRISTIAN " <cristian. @proys.unirioja.es> To: <jmurillo@dmc.unirioja.es>,

Hola: Le envio las respuestas a las cuestiones planteadas en la actividad A8 en el fichero de Cabri. Queria comentarle que en la pagina del proyecto solo aparecen activadas las cuatro primeras preguntas, por lo que faltan esta y todas las demas preguntas. Espero que se solucione y podamos continuar con la actividad A9.

Un saludo: Cristian

\subsection{CATEGORÍAS ALUMNO/CONTENIDO}

Establecemos las siguientes categorías de las relaciones de los alumnos con el contenido, que nos permiten determinar la comprensión de los contenidos presentados por el profesor(curriculum implementado).

ARE: Los alumnos RESPONDEN. Los alumnos dan respuestas a los problemas o actividades propuestas.

\section{Enviado por Cristian:}

Me sugiere que el triangulo grande esta formado por 3 triangulos. Y que las areas de estos triangulos sumadas dan la del grande, ademas las alturas sumadas tambien. Esto lo demuestro por los siguiente:

$$
\begin{gathered}
A^{\prime}+A^{\prime \prime}+A^{\prime \prime \prime}=A G \\
b^{\prime *} h^{\prime} / 2+b^{\prime \prime *} h^{\prime \prime} / 2+b^{\prime \prime \prime} * h^{\prime \prime \prime} / 2=h * b / 2 \\
\text { Simplificando: } h^{\prime}+h^{\prime \prime}+h^{\prime \prime \prime}=h
\end{gathered}
$$

ACO: Los alumnos COMPRENDEN. Envían mensajes o textos que manifiestan su comprensión de los contenidos.

\section{Respuesta a romcuad.}

From: “CRISTIAN “ cristian@proys.unirioja.es To: <jmurillo@dmc.unirioja.es>, 
Hola: He recibido la respuesta a la solución de la cuestión del rombo. He visto las nuevas preguntas que proponia y he modificado las soluciones aclarandolas.

Un saludo: Cristian

AEX: Los alumnos EXPLICAN. Establecen conclusiones sobre lo tratado, conectan las nuevas ideas con otras anteriores, expresan de modo organizado su dominio del contenido, hacen propuestas para avanzar en el contenido o para aplicarlo a situaciones problemáticas.

Re: Problema 3 modificado.

Enviado por Cristian: En contestación a: Problema 3 enviado por Cristian:

Dado un triangulo equilatero con un punto $P$ en su interior, y este punto lo unes los vertices del triangulo resultan tres triangulos cuyas alturas son las distancias desde el punto $P$ hasta los lados del triangulo equilatero.

La suma de las areas de estos tres triangulos nuevos, es igual al area del triangulo equilatero, porque estos estan dibujados en su interior. Si la formula del area es base por altura dividido entre dos. Se deduce que a partir de la siguiente ecuacion:

$$
\begin{aligned}
& A=A^{\prime}+A^{\prime \prime}+A^{\prime \prime \prime} \\
& (\text { Báh }) / 2=\left(B^{\prime} a ́ h^{\prime}\right) / 2+\left(B^{\prime \prime} \text { áh" }\right) / 2+\left(B^{\prime \prime \prime}{ }^{\prime \prime} h^{\prime \prime \prime}\right) / 2 \\
& \text { Báh=B'áh'+B"'áh" }+B^{\prime \prime \prime} h^{\prime \prime \prime} \\
& \text { Báh=Báh'+Báh"'+Báh"' } \\
& \text { Báh=Bá }\left(h^{\prime}+h^{\prime \prime}+h^{\prime \prime \prime}\right) \\
& h e=\left[B a ́\left(h^{\prime}+h^{\prime \prime}+h^{\prime \prime}\right)\right] / B \\
& h e=h^{\prime}+h^{\prime \prime}+h^{\prime \prime}
\end{aligned}
$$

Como es un triangulo equilatero sus lados son iguales, por lo que las bases de todos los triangulos son iguales. Se sustituye y sale como resultado que la altura del triangulo equilatero es igual a la suma de las alturas de todos los triangulos dibujados en el interior del grando.

ACN: Los alumnos CONJETURAN. Establecen hipótesis sobre lo tratado o sobre algún resultado. Hacen conjeturas de generalización sobre algún resultado particular.

\section{Solución pregunta:}

From: "CRISTIAN " cristian@proys.unirioja.es To: <jmurillo@dmc.unirioja.es>,

Hola: Le envío las soluciones que se me han ocurrido a su pregunta.

Un saludo: Cristian

ADE: Los alumnos DEFINEN. Dan enunciados o recogen en forma de teorema o proposición los resultados obtenidos en la resolución de algún problema o como respuesta a alguna cuestión.

\section{De nuevo respuesta.}

From: "CRISTIAN “ cristian@proys.unirioja.es To: <jmurillo@dmc.unirioja.es

Hola: De nuevo le envio las respuestas a la cuestión que nos planteo sobre el rombo y que de nuevo nos plantea. Envio mis conclusiones en el fichero adjunto de Cabri. Espero que esta vez sean lo suficientemente concisas y explicativas.

Un saludo. Cristian 
Consideramos también las dos siguientes categorías, que aunque no se han dado en los casos considerados, en el análisis si que han aparecido con otros alumnos y pensamos que puede ser de interés citarlos aquí.

APR: Los alumnos PREGUNTAN. Quieren que se les facilite la comprensión del contenido: No entiendo que quiere decir cortar por la diagonal y volver a pegar de otra forma.

AVL: Los alumnos VALORAN. Enjuician los argumentos y contenidos de las actividades propuestas y toman posiciones al respecto: lo que dice Vanesa no es cierto porque...

Como resumen del funcionamiento del entorno interactivo de aprendizaje proponemos el siguiente gráfico-esquema:

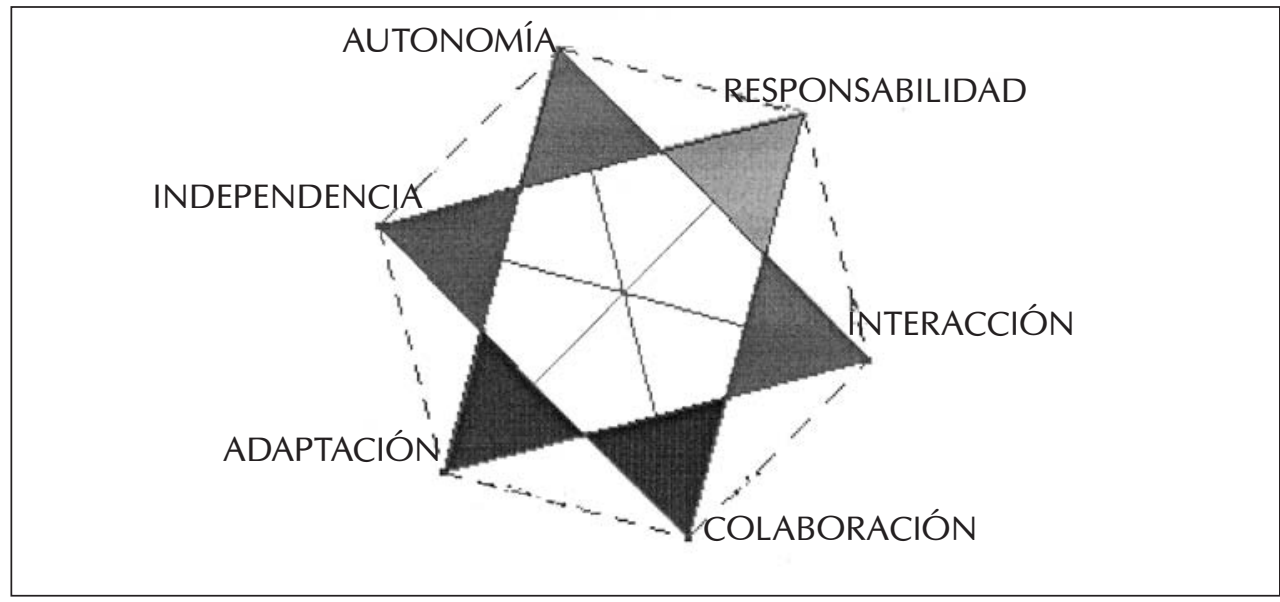

Figura 4. Funcionamiento del entorno interactivo.

\section{METODOLOGÍA DE INNOVACIÓN}

Los contenidos se han seleccionado teniendo en cuenta que el Taller de Matemáticas de $4^{\circ}$ de ESO es una asignatura optativa de dos horas semanales de clase. Las actividades se han construido, por una parte con la idea de que sean autosuficientes y por otra que potencien el espíritu de confianza en el profesor y la colaboración y ayuda entre los alumnos, además de conseguir el aprendizaje de los contenidos geométricos correspondientes. Todas las actividades, están enmarcadas en el programa de Geometría de $4^{\circ}$ de la E.S.O. y se presentan con varias cuestiones de distintos niveles de dificultad y profundidad. Llevan incorporadas ayudas progresivas, que facilitan al alumno la realización de la actividad utilizando el programa CABRI II. Para solicitar otro tipo de ayuda a los profesores o a otros compañeros pueden hacerlo a través del correo electrónico, mediante un enlace con la lista de las direcciones electrónicas de los participantes. Las sucesivas respuestas (réplicas) a las cuestiones planteadas, pueden enviarse al profesor(según se haya planificado) o al forum para ser replicada por los distintos participantes. Las diversas acciones de tutorización, aparecen como puntos de interactividad en las actividades planteadas. 


\begin{tabular}{|l|l|l|l|l|l|l|l|l|}
\hline $\begin{array}{l}\text { Palabras } \\
\text { clave }\end{array}$ & Ayuda & $\begin{array}{l}\text { Más } \\
\text { ayuda }\end{array}$ & $\begin{array}{l}\text { Ayuda } \\
\text { en línea }\end{array}$ & Sugerencia & Facilita & $\begin{array}{l}\text { Consultar las } \\
\text { respuestas }\end{array}$ & $\begin{array}{l}\text { Ampliación } \\
\text { "TEOREMA } \\
\text { DE TU NOMBRE }\end{array}$ & $\begin{array}{l}\text { Ayudas } \\
\text { técnicas }\end{array}$ \\
\hline
\end{tabular}

\section{Problema 3}

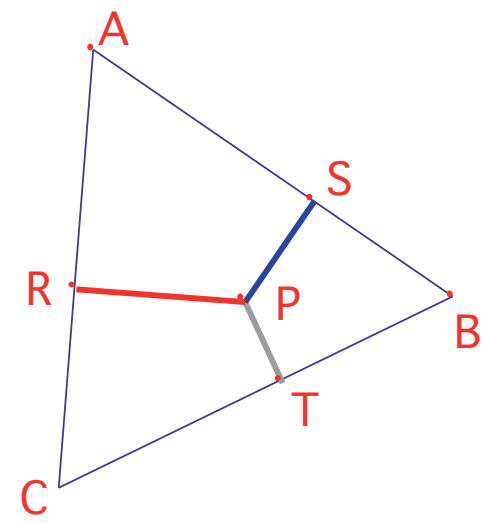

Sea $\mathbf{A B C}$ un triángulo equilátero cualquiera -es importante que el triángulo que construyas con Cabri se mantenga siempre equilátero-. Sea $\mathbf{P}$ un punto interior del triángulo $\mathbf{A B C}$. Determinar las distancias PS, PR y PT, desde el punto $\mathbf{P}$ a cada uno de los tres lados del triángulo.

Nota: si tienes problemas para determinar la distancia de un punto a una recta haz click en el texto en azul y subrayado distancias.

1. Suma las longitudes de los segmentos PS, PR y PT. (utiliza la calculadora de Cabri).

2. Elige otro punto $\mathbf{P}^{\prime}$ también interior al triángulo $\mathbf{A B C}$. Realiza las mismas operaciones que con el punto $\mathbf{P}$ del apartado anterior. Compara el resultado de la suma de este apartado con la obtenida en el apartado 1.

3. ¿La comparación de los resultados del apartado anterior te sugiere alguna idea? Por favor piensa un poco y reflexiona -es muy importante-. Si no se te ocurre nada haz click en ayuda. Si después de haber utilizado ayuda todavía no te sugiere alguna idea puedes consultar más ayuda.

¿Qué crees que pasar si se modifica el tamaño del triángulo equilátero?. Haz una conjetura sobre la suma de distancias de un punto interior a los lados del triángulo.

Figura 5. Acciones de tutorización.

\section{METODOLOGÍA DE LA INVESTIGACIÓN: INDAGACIÓN Y EVALUACIÓN}

Con los planteamientos e instrumentos (categorías, elementos de la interacción electrónica e indicadores) que hemos establecido en el marco teórico (Murillo, J., 2001) y los conceptos de Momento y de Episodio, que definimos a continuación, procederemos a un análisis, estudio y evaluación de la naturaleza de las interacciones que aparecen, cuando un grupo de cinco alumnos desarrollan trabajo colaborativo en la resolución de actividades.

Definimos Momento 0 como el intervalo de tiempo en el que tiene lugar el planteamiento de la actividad. 
Definimos Momento $i$ como el intervalo de tiempo en el que tiene lugar una acción generada por la que ha tenido lugar en el Momento i-1.

Definimos Episodio como el conjunto de Momentos que han tenido lugar a partir de un Momento 1.

Para el estudio de las interacciones tendremos en cuenta las relaciones entre los distintos componentes del entorno interactivo, -ecosistema de aprendizaje- que nos permitirán determinar la naturaleza de las mismas. Atendiendo a los elementos de la interacción electrónica y a los indicadores, podremos determinar la efectividad de las interacciones y señalar la influencia de las mismas en el aprendizaje.

Analizamos un forum que se desarrolla con una de las dos metodologías utilizadas: planteamos previamente un problema en la página de actividades y una vez que han respondido a la cuestión propuesta, les planteamos que justifiquen y determinen por qué se da una determinada propiedad para ser discutida y replicada por todo el grupo en el forum(en la segunda metodología: se plantea una pregunta mediante un mensaje general a todos los alumnos que sirve de elemento de discusión y a partir de la cual se desarrolla el forum).

\subsection{ANÁLISIS DEL DISCURSO EN EL FORUM DE EDUARDO}

\section{Subject: pregunta para discutir: T.Inscrito. Date: Mon, 24 May 1999 11:16:49 From: jmuri- Ilo@dmc.unirioja.es To: Proyecto Clavijo}

Mis queridos amigos: He mirado la página del tablero electrónico y está totalmente despejada. He pensado que sería bueno que la animásemos un poco con nuestras opiniones sobre algún tema. La pregunta sobre la que opinar podía ser la siguiente:

" Dado un triángulo equilátero, se inscribe en él otro triángulo equilátero, ¿qué pasa con el área del triángulo inscrito según se mueven sus vértices a lo largo de los lados del triángulo de partida?"

Podéis plantear las dudas sobre cualquier aspecto del enunciado o sobre cualquier respuesta dada, a cualquiera de los miembros del Forum. Las respuestas hay que enviarlas al Tablero. Podéis utilizar Cabri para haceros una composición de lugar sobre la cuestión planteada.

Saludos y adelante. Jesús

ARE, AEX.Enviado por EDUARDO en fecha Mayo 24, 1999 a las 13:34:39:

El área del triángulo inscrito es mínima cuando sus vértices están situados en el punto medio de los lados del triángulo inicial, se va haciendo más grande a medida que se acercan los vértices del triángulo inscrito a los vértices del triángulo inicial. El área mínima es 1/4 de la total. El área máxima es todo el triángulo inicial [Respuesta Activa] 


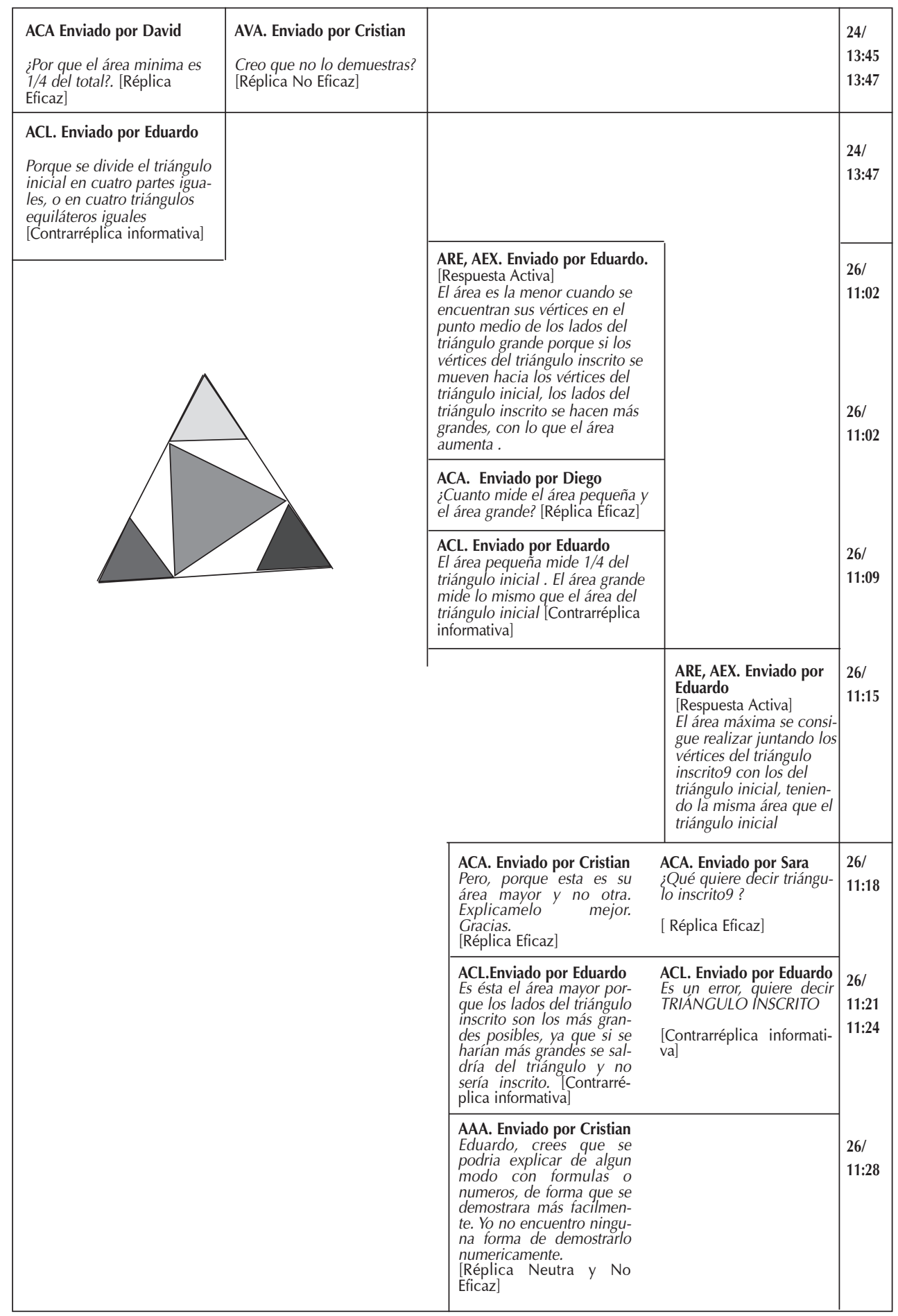

Figura 6. Análisis del discurso en el forum de Eduardo. 


\section{EFICACIA.}

Efectividad de las interacciones en el forum correspondiente a TRIÁNGULO INSCRITO de Eduardo: Interacción positiva.

Indicador 8 (respuesta aceptable con demostración incompleta, respuesta correcta con demostración incompleta).

En el forum de Eduardo se distinguen tres episodios: Episodio 1, desarrollado durante el primer día, Episodio 2, desarrollado en la primera parte del segundo día y Episodio 3, desarrollado en la segunda parte del segundo día.

Consideramos que globalmente la interacción ha sido positiva, pues aunque la demostración y justificación sigue siendo incompleta, si que con las aclaraciones dadas por Eduardo y las aportaciones de sus compañeros en el desarrollo del forum motivado en torno a "Pregunta para discutir: Triángulo inscrito" se ha producido una mejora en la respuesta.

En el Episodio 1, Eduardo responde de forma global a la pregunta planteada, explicando la evolución de las áreas y dando los valores máximo y mínimo del área del triángulo inscrito, pero no justifica adecuadamente estos valores. Cristian valora la respuesta de Eduardo, pero no queda claro que solicite algún tipo de aclaración, por lo que posiblemente esta es la causa de no recibir respuesta. A David le responde de forma adecuada, aclarándole la cuestión planteada.

En el Episodio 2, Eduardo responde de nuevo con la misma respuesta dada en el Episodio 1. A la pregunta planteada por Diego en su réplica, Eduardo responde pero en la práctica no la justifica.

En el Episodio 3 del forum, Eduardo responde de nuevo parcialmente a la pregunta planteada y explica cuando el área del triángulo inscrito es máxima, pero tampoco da una justificación completa. El diálogo con Sara se limita a aclarar una duda de carácter tipográfico. El diálogo con Cristian es largo y fluido y supone una mejora en la respuesta, se finaliza con una expresión de ánimo para continuar.

\section{PERFIL DE APRENDIZAJE DE EDUARDO}

En opinión del profesor presencial, responsable de la asignatura de Matemáticas de $4^{\circ}$ de la E.S.O., las características de Eduardo responden a las de un alumno con nivel medio en relación al resto de la clase. El perfil de aprendizaje de Eduardo es bastante complejo, ya que se presentan tres Episodios, en el primero a su vez se distinguen dos líneas (interacciona con dos compañeros), en el segundo episodio interacciona con un único compañero y en el tercer episodio interacciona con dos compañeros por lo que aparecen dos líneas.

Gráfico interactivo del perfil de aprendizaje de Eduardo. 


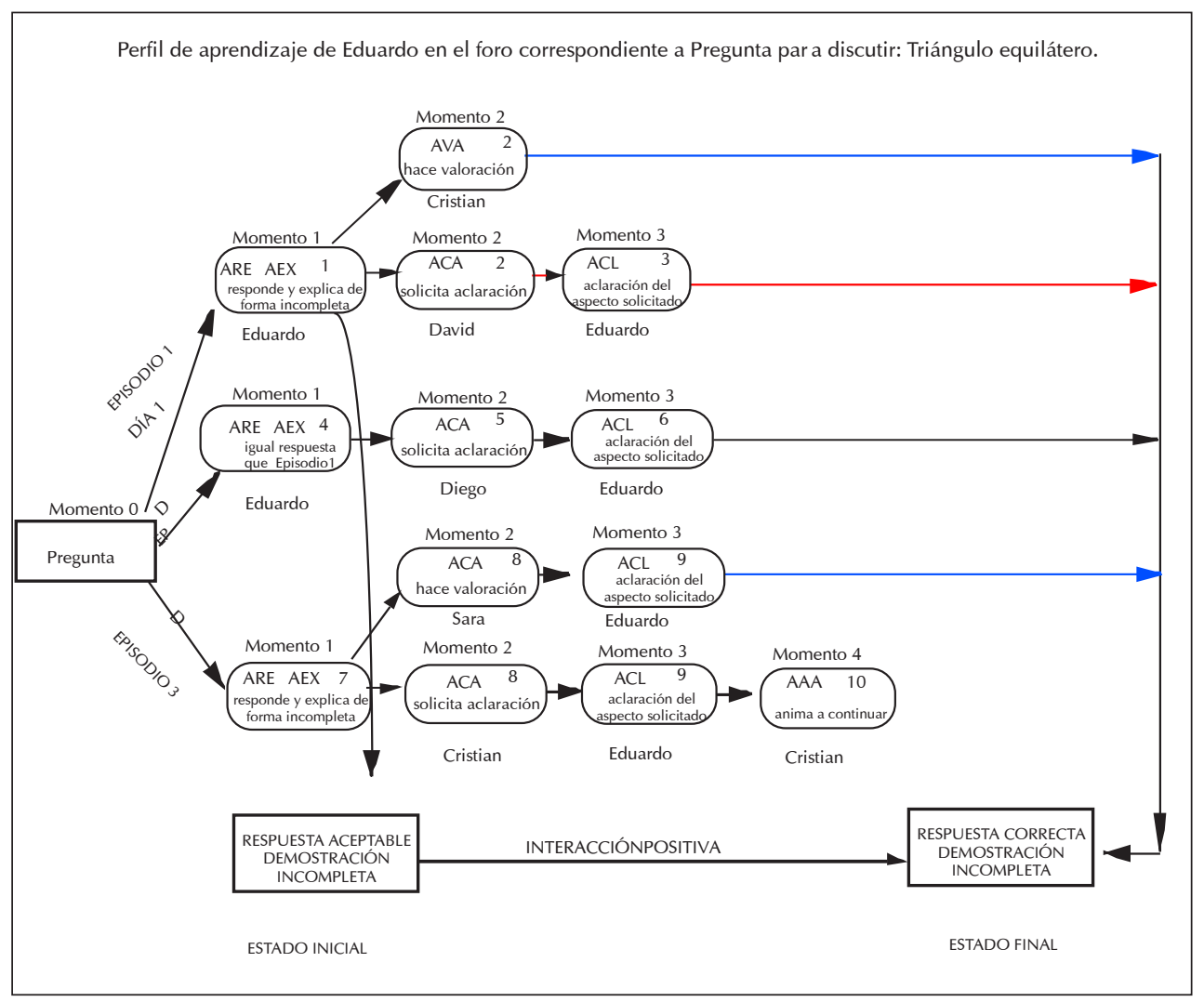

Figura 7. Perfil de aprendizaje de Eduardo.

Consideramos que globalmente la interacción es positiva, puesto que las interacciones con los compañeros, las réplicas pidiendo clarificación de las respuestas, las aclaraciones dadas, las sugerencias y valoraciones de las respuestas, han perfeccionado la respuesta original, aunque la demostración no es completa.

Para un mejor seguimiento, del perfil de aprendizaje, dado la complejidad del mismo, hemos numerado las acciones de forma cronológica.

\subsection{BENEFICIOS Y EVOLUCIÓN CON RESPECTO A OTROS DEL CONOCIMIENTO DE EDUARDO}

Estructuramos esta parte del análisis del forum en: estado inicial, primera respuesta, visión del alumno, beneficios cognitivos, nuevos enfoques y resumen.

\section{Estado inicial}

Según las informaciones previas, que teníamos de Eduardo, relatadas por su profesor presencial, conocemos que Eduardo tiene un cierto grado de familiaridad con los conceptos previos del problema del T.inscrito, necesarios para iniciar su exploración, como son los de Triángulo equilátero, inscrito en otro, la idea de movimiento de un vértice a lo largo de un lado, área de un triángulo... Quizá la idea inicial de inscripción estaba mediatizada por la consideración sólo del triángulo que une los 
puntos medios, concepción que es bastante común a los alumnos de su edad e incluso superiores. En palabras de su profesor presencial decía de Eduardo, que en comparación con el resto de la clase tenia un "nivel medio".

\section{Primera respuesta}

Puesto que pretendemos conseguir una mejora en la comunicación matemáticas, consideramos que la respuesta inicial de Eduardo al problema planteado es aceptable. Si considerásemos simplemente el nivel de conocimientos correspondiente a $4^{4}$ de la ESO, podríamos admitir la respuesta como completa. En el resto del protocolo, Eduardo explica y matiza a sus compañeros (ante sus preguntas) lo que ha dicho en su primera intervención. En términos de justificación (o demostración), posiblemente poco más se puede esperar de un alumno de su edad. Es muy frecuente dar la justificación de 1/4 recortando el triángulo pequeño y superponiéndolo con los otros tres que salen para visualizar su igualdad. Con Cabri es posible hacer una comprobación parecida pero más dinámica.

\section{Visión del alumno}

Las respuestas de Eduardo a las preguntas de David y Cristian, si consideramos la respuesta textual, relaciona de forma incorrecta el área con la longitud de los lados (mayor longitud mayor área), salvo en el contexto de los triángulos equiláteros, que consideramos que es al que se refiere aunque no lo diga explícitamente. Eduardo insiste en esta idea de relación lado-área en la penúltima intervención del protocolo del forum. Esto hace que podamos interpretar la idea que Eduardo tiene de triángulo inscrito como el que no se puede salir del original (ligada a la idea de longitud de los lados).

\section{Beneficios cognitivos}

Los beneficios cognitivos que se apropia del espacio social, que constituye este forum, los podemos interpretar en términos de explicitación y profundización de los contenidos, que han ido apareciendo relacionados con: La división del triángulo original en cuatro triángulos equiláteros, la relación lado-área en triángulos equiláteros y la idea de triángulo inscrito (relacionado con la del movimiento de los vértices sobre los lados del triángulo original.

Nuevos enfoques

No se detecta ningún nuevo enfoque del problema usando las ideas de semejanza, transformación o variación continua del área como función. Quizás la insistencia de Cristian solicitando una demostración numérica o con fórmulas, suponga una petición o introducción en el diálogo de un elemento nuevo: un tipo de razonamiento métrico y/o algebraico, el de demostración numérica o con fórmulas. Aspecto este que no ha aparecido antes, ni siquiera en el enunciado del problema, creyendo quizás, este último que esa es la respuesta que podría satisfacer al profesor virtual, por la costumbre en la clases de matemáticas en usar este tipo de razonamientos algebraicos.

\section{Resumen}

En resumen el forum como espacio social de interacciones. ha conseguido mejorar la respuesta de Eduardo aumentando su grado de significación del área del triángulo inscrito y la relación de esta con la longitud de sus lados. 


\section{REFLEXIONES Y CONCLUSIONES}

Presentamos aquí una serie de reflexiones y conclusiones, en base al análisis de los datos recogidos en la investigación, tanto del forum, como de los mensajes de correo electrónico, y la información aportada por el profesor presencial.

Se ha conseguido una utilización correcta y adecuada del soporte instruccional, aunque es necesario señalar la necesidad de dedicar un tiempo suficiente para que los alumnos lleguen a dominar con soltura el uso simultáneo del navegador, el correo electrónico y CABRI II. Hay que tener en cuenta además el doble sistema de trabajo utilizado, en el que la participación puede hacerse: unas veces en el tablero electrónico de respuestas (Forum) que tiene su dinámica particular de respuestas, réplicas y contrarréplicas y otras a través del correo en el que deben además adjuntar su archivo de CABRI II. En caso contrario, podría ocurrir que el atractivo y posibilidades del medio tropezasen con las dificultades de la propia herramienta a la hora de trabajar con ella. De hecho al principio durante las clases, una parte del tiempo se ha dedicado a resolver aspectos de funcionamiento del navegador, el correo o el foro de discusión, pese a que previamente se dedicó un tiempo que aparentemente era suficiente para que los alumnos se familiarizaran con su manejo. No se puede dar por sentado, por tanto, que los alumnos aprenden a manejar cualquier programa en poco tiempo y sin ninguna dificultad. Es necesario darles el tiempo suficiente no sólo para que los utilicen correctamente, sino para que interioricen la forma especial de trabajar con ellos y sepan emplearlos adecuadamente.

Aunque, en principio, el sistema de enseñanza aprendizaje se desarrolla de una forma más lenta que el sistema tradicional presencial, puesto que se exige que las respuestas sean respondidas con una expresión adecuada y con mayor rigor, consideramos que los contenidos se aprenden de un forma más significativa y en definitiva más rentables en términos educativos, potenciando la comunicación matemática, la institucionalización de los resultados obtenidos, el aprendizaje colaborativo, y en general desarrollando su expresión y comunicación y facilitando el dominio de la Red, que les acerca más al perfil del alumno del siglo XXI. La experiencia de contacto escrito, a través del correo electrónico ha sido muy positiva para todos, abarcando su influencia no sólo el ámbito instructivo, con ramificaciones en distintas áreas, sino que entra dentro del ámbito puramente educativo del respeto hacia los demás, de la cortesía en el trato, de las buenas maneras, de la importancia del trabajo bien hecho y la expresión clara, en fin, de esa "educación" en el sentido más amplio.

En cuanto a las actividades que se plantean a los alumnos, deben coexistir los dos tipos que nosotros hemos utilizado, por una parte unas, al comienzo, enfocadas fundamentalmente al aprendizaje del programa y del entorno interactivo; atractivas y que tengan enlaces de ayuda, de manera que puedan ser resueltas por los alumnos casi de forma autónoma y otras, con posterioridad más abiertas, con la finalidad de posibilitar a los alumnos la utilización de su imaginación, su creatividad y la libertad y en definitiva potenciar la actividad matemática. Las actividades abiertas suponen un esfuerzo importante a nuestros alumnos, y a veces están desorientados, no saben qué camino elegir y solicitan bastantes orientaciones, pero los resultados obtenidos y la satisfacción encontrada al responder, muestran que son necesarias y útiles.

Un aspecto interesante en relación a las características del entorno diseñado y utilizado, es el hecho de que permite dirigirse a una población heterogénea en cuanto 
a la edad y su cualificación -frente a la enseñanza puramente presencial que en general va dirigida a una población homogénea, al menos en cuanto a la edad-, pudiendo trabajar con alumnos de diferentes edades, niveles cognitivos y capacidades, aunque resulta claro que para los alumnos con necesidades educativas especiales, hay que diseñar actividades específicas. El aprendizaje se plantea en una situación de mayor libertad frente a una situación controlada, generalmente en un contexto de dependencia.

Un aspecto, que podemos considerar negativo, en relación al soporte utilizado, se encuentra relacionado con la evaluación. Es difícil detectar el fraude, por lo que se plantean problemas de validez, haciendo que se reduzca su credibilidad.

En relación con el profesorado y la metodología a utilizar, es necesario destacar, que la utilización de muy diversos medios tecnológicos y de comunicación requiere una preparación específica de los docentes, producción de material y actividades específicas y bien elaboradas, que sirvan para entornos, niveles y estilos diversos de aprendizaje y que permitan a los alumnos ritmos propios de aprendizaje, dándoles autonomía suficiente para programar su ritmo y forma de aprender, y con la idea central de considerar al alumno centro del proceso de aprendizaje y en sujeto activo de su formación. El material didáctico se puede estructurar de manera que sea posible la autoevaluación, con lo que de motivador tiene conocer con inmediatez los progresos del propio aprendizaje. No obstante, hay que señalar las limitaciones de este tipo de enseñanza para alcanzar aquellos objetivos que no atienden a capacidades que se expresen por escrito, que en gran parte podrían ser obviados con una utilización adecuada y complementaria de la teledifusión y videoconferencia de grupo.

En relación a los beneficios generados por la utilización del soporte diseñado, el análisis de los resultados correspondiente a las interacciones que se han producido, confirma la idea de que la construcción social del conocimiento constituye un método valiosísimo de enseñanza y reafirma la importancia del medio y de los procesos que se ponen en juego en la institución escolar para conseguir el aprendizaje. Las interacciones entre iguales han jugado un papel fundamental para facilitar y acelerar ciertas adquisiciones de destrezas, habilidades y conocimientos. A la vista de los resultados parece claro que la utilización de este soporte, entorno interactivo de aprendizaje de la geometría -ecosistema de aprendizaje- ha potenciado el aprendizaje y una actitud más positiva hacia la Geometría y en consecuencia el que nuestros alumnos tengan un mayor éxito en sus estudios.

Dentro del entorno de aprendizaje diseñado, cabe señalar que en el Tablero electrónico las interacciones que se han producido son multidireccionales entre todos los participantes en el foro de discusión, en el que podemos considerar que el profesor es uno más, con la salvedad de ser el responsable de plantear las cuestiones a resolver y organizar el foro. Sin embargo en las actividades propuestas y realizadas utilizando el correo electrónico como medio de comunicación, la interacción ha sido bidireccional.

En el primer caso -Tablero- podemos hablar de trabajo colaborativo en la resolución de problemas, los alumnos en su relación con otros compañeros participan de algunas de las funciones del profesor. En las interacciones establecidas a través del correo electrónico podemos considerarlo más bien en una enseñanza y tutoría personalizada. 
Tanto en un caso como en el otro, a través de las preguntas planteadas bien por el profesor bien por otro compañero se ha conseguido una participación activa de los alumnos en su aprendizaje y construcción del conocimiento.

El papel de los alumnos ha cambiado pasando a ser parte activa en la construcción de su conocimiento y en el de sus iguales. También el rol del profesor ha cambiado, en el sentido de no ser la única fuente de conocimiento, sino que más bien ha actuado como guía de las discusiones de los alumnos, facilitando y ayudándoles a lo largo del proceso de aprendizaje, mediante las sugerencias y ayudas oportunas. El control del entorno de aprendizaje se ha mostrado como un aspecto también fundamental del papel del profesor.

\section{BIBLIOGRAFÍA}

ALVAREZ, A. y otros. (1997). Presentación: Lo actual y lo potencial en la Zona de Desarrollo de la educación española. C \& E : Cultura y Educación, 6-7.

ARNOLD, S. et al. (1996). Critical Issues in the Distance Teaching of Mathematics and Mathematics Education. En BISHOP, A.J. y otros (eds.), International Handbook of Mathematics Education. Dordrech: Kluwer Academic Publisher.

BALACHEFF N. y KAPUT, J. (1997). Computer-Based Learning Enviroments in Mathematics. En A.J. BISHOP y otros (eds.), International Handbook of Mathematics Education. Dordrech: Kluwer Academic Publisher.

BALACHEFF, N. (1994). Didactique et inteligence artificiell. Recherches en Didactique des Mathematques, 14 (12), 9-42.

BARBERA, E. (2000). Study actions in a virtual university. Virtual University Journal, $3(2), 31-42$

BROWN, A.L. y PALINCSAR, A.S. (1982). Inducing strategic learning from text by means of informed, self-control training. Topics in learning and Learning Disabilities, 2, 1-17.

BRUNER, J. (1978). The role of dialogue in language acquisition. The child's conception of language. New York: Springer-Verlag.

BRUNER, J.(1996). The Culture of Education. Harvard University.

COBB, P. y BAUERSFELD, H. (Eds.).(1995). The emergence of Mathematical Meaning: Interaction in Classroom Cultures. Hillsdale: Lawrence Erlbaum Associates.

COBO, P. y FORTUNY, J.M. (2000). Social Interactions and Cognitive Effects in Contexts of Area-Comparison Problem Solving. Educational Studies in Mathematics, 42, 115-140.

COLE, M. (1996). Cultural psychology a one and future discipline. Harvard University. CROOK, C. (1998). Ordenadores y aprendizaje colaborativo. Madrid: Morata, MEC. DILLENBOURG, P. (ed.) (1999). Collaborative learning: cognitive and computational approaches. Amsterdam: Pergamon.

DUART, J.M. (2000). Aprendizaje sin distancias. [documento electrónico: http://campus.uoc.es/web/cat/articles/josep_maria_duart.html]. Barcelona: UOC.

FORTUNY, J.M. y otros (1999). Aprendizaje sin límites. Un modelo de diseño interactivo como soporte y ampliación instruccional en la enseñanza de la geometría en la ESO. Contextos Educativos: Revista de Educación, 2, 27-52 
FRASER, S. y DEANE, E. (1999). Educating Tomorrow's Scientists: IT as a tool, not an educator. Teaching in Higher Education, 4 (1).

GAIRÍN, J.M. (1998). Sistemas de representación de números racionales positivos. Un estudio con maestros en formación. Tesis doctoral. Departamento de matemáticas. Universidad de Zaragoza.

GRAHAM, P. (ed.) ( 1999). Teacher/mentor : a dialogue for collaborative learning. New York : Teachers College Press.

HERSHKOWITZ, R. y SCHWARZ, B. (1999). The emergent perspective in rich learning environments: some roles of tools and activities in the construction of sociomathematical norms. Educational Studies in Mathematics, 39, 149-166.

JÄRVELÄ, S. (1996). New models of teacher-student inteaction: A critical review. European Journal of Phychology of Education, XI (3), 249-268.

JOHNSON, D.W. y otros (1999). El Aprendizaje cooperativo en el aula. Buenos Aires: Paidós.

KRUGER, J. (ed.) (2001). Computers in the delivery of special education and related services, developing collaborative and individualized learning environments. Binghamton, NY: Haworth Press.

LOBATO, C. (1998). El trabajo en grupo: aprendizaje cooperativo en secundaria. Universidad del País Vasco, Servicio Editorial.

MURILLO, J. (2001). Un entorno interactivo de aprendizaje con Cabri-actividades, aplicado a la enseñanza de la geometria en la E.S.O. Tesis doctoral Universitat Autònoma de Barcelona.

PALINCSAR, A.S , BROWN, A.L. (1984). The reciprocal teaching of comprehension monitoring activies. Cognition and Instruction, 1, 117-175.

PERRET-CLERMONT, A. (1984). La construcción de la inteligencia en la interacción social : aprendiendo con los compañeros. Madrid: Visor.

ROGOFF, B. (1994). Developing understanding of idea of communities of learners. Mind, Culture and Activity, 1(4), 209-229.

SOURY-LAVERGNE, S. (1998). Etayage et explication dans le preceptorat distant, le cas de TeleCabri. Tesis doctoral. Université Josep Fourier. UFR Informatique et Mathematiques appliques, Grenoble.

SERRANO, J.M. (1997). Aprendizaje cooperativo en matemáticas : un método de aprendizaje cooperativo-individualizado para la enseñanza de las matemáticas. Universidad de Murcia.

SLAVIN, R.E. (1999). Aprendizaje cooperativo: teoría, investigación y práctica. Buenos Aires: Aique. 